\title{
Increased Responsiveness to Synthetic Thyrotropin-Releasing Hormone (TRH) in Androgenized Adult Rats with Special Reference to the Dose of Testosterone Propionate for Androgenization
}

\author{
Tомоко FUJII $^{1}$, Junzo KATO ${ }^{2}$, AND KATsumi WAKABAYASHI ${ }^{3}$ \\ ${ }^{1}$ Department of Pharmacology, and ${ }^{2}$ Department of Obstetrics \\ and Gynecology, Teikyo University School of Medicine, \\ Tokyo 173, and ' ${ }^{3}$ Institute of Endocrinology, \\ Gunma University, Maebashi 371, Japan
}

\begin{abstract}
Synopsis
Five-day-old female rats were androgenized with a single dose of $100 \mu \mathrm{g}$ (TP100A) or $1000 \mu \mathrm{g}$ (TP1000A) testosterone propionate. The androgenized adult rats together with normal rats were tested for prolactin and TSH responses to synthetic TRH. Five $\mu \mathrm{g} / \mathrm{kg}$ of $\mathrm{TRH}$ was injected iv 4 times at $2 \mathrm{hr}$ intervals, and serum samples were obtained $5 \mathrm{~min}$ after each injection. The prolactin release by exogenous TRH in normal rats was less marked. In TP1000A rats, the basal prolactin level in the serum was higher than that in normal or TP100A rats and a further elevation was induced by the 1 st injection of TRH. In TP100A rats, the serum prolactin level increased five-fold more than the maximal level in normal rats by the 1 st and 2 nd injections, while the pituitary prolactin content showed a less marked decline and a tendency to increase after the 4th injection, suggesting an enhanced release and production of prolactin by TRH in TP100A rats. TRH injection stimulated TSH release in androgenized rats as well as in normal rats. The magnitude in the TSH release was TP1000A $>$ normal $\geqq$ TP100A. The TSH contents in the pituitary after TRH injection tended to decrease in TP1000A and normal rats but not in TP100A rats. In TP100A rats, increases of TSH content were evident by the $3 \mathrm{rd}$ and 4 th TRH injections. The weight of the anterior pituitary decreased in normal and TP1000A rats but tended to increase in TP100A rats after the treatment with TRH. The present results indicate that not only lactotrophs but also thyrotrophs in the pituitary became very sensitive to exogenous TRH by neonatal androgenization. A marked difference in the responsiveness to exogenous TRH between TP100A and TP1000A rats could be due to the fact that the alterations in the hypothalamus and secondarily in the ovary were induced by testosterone propionate in the dose-dependent manner.
\end{abstract}

Androgenized female rats possessed an increased responsiveness to synthetic LHRH during the maturation particularly at around 7 weeks of age and a reduced responsiveness to LHRH after they became mature as compared with normal control rats (Fujii, et al., 1978). The dose of testosterone propionate (TP) used for androgenization has been found to affect markedly the pituitary function. Rats androgenized with

Received October 19, 1978.
$100 \mu \mathrm{g}$ TP showed a much greater LH release after iv injection of LHRH than rats androgenized with $1000 \mu \mathrm{g}$ TP when tested at 7 weeks old (Fujii, et al., 1978).

A marked increase in the serum prolaction level after 7 weeks of age has been noted in the rats androgenized with 1000 $\mu \mathrm{g}$ TP (Fujii, et al., 1974), suggesting an increased production of prolactin in the pituitary or altered responsiveness of the pituitary to prolactin-inhibiting factor (PIF) and/or to prolactin-releasing factor (PRF). 
Synthetic TRH has been demonstrated to induce the rapid release of prolactin in addition to TSH in man (Jacobs, et al., 1971) and in other animals (Tashjian, et al., 1971). Therefore the present experiment was undertaken to examine the prolactin response to synthetic TRH in adult female rats androgenized neonatally with $100 \mu \mathrm{g}$ or $1000 \mu \mathrm{g}$ of $\mathrm{TP}$ in order to know the ability of the pituitary to synthesize and release prolactin. In addition, TSH response to TRH was analyzed in these animals.

\section{Materials and Methods}

Wistar-Imamichi strain female rats received a single dose of $100 \mu \mathrm{g}$ (TP100A) or $1000 \mu \mathrm{g}$ (TP1000A) testosterone propionate dissolved in $0.05 \mathrm{ml}$ olive oil on the 5th day of life. Control animals received olive oil alone. They were kept in an air conditioned room $\left(22 \pm 2^{\circ} \mathrm{C}, 55 \pm 5 \%\right.$ humidity $)$ with a lighting schedule of 14 hr light ( $6: 00$ a.m. $-8: 00$ p.m.) and $10 \mathrm{hr}$ darkness, and supplied with a stock diet (Oriental Yeast Co.) and water ad libitum. The onset of persistent estrus in TP1000A rats varied between 28 days old and 40 days old, whereas in TP100A rats, 42 percent were in estrus at 32 days old and 100 percent at 65 days old. Normal control rats were used at the stage of estrus. Synthetic TRH (Sankyo Pharmaceutical Co.) was dissolved in saline solution to give a concentration of $5 \mu \mathrm{g} / \mathrm{m} l$. Twelve to 13-week-old normal rats, TP100A and TP1000A groups were injected with $5 \mu \mathrm{g} / \mathrm{kg}$ TRH iv 4 times every $2 \mathrm{hr}$ starting at $11: 00$ a.m. into a branch of saphenous vein at a hind limb without anesthesia to avoid the effects of anesthesia on prolactin secretion (Wakabayashi et al., 1971). Four to 6 animals were killed by decapitation $5 \mathrm{~min}$ after each injection. In a preliminary experiment, no significant effect of a single or 4 injections of saline solution on serum prolactin or TSH levels was found. Therefore, 6 control rats of each group were killed around 11:00 a.m. without saline injection to determine the basal levels of prolactin and TSH. The blood samples were kept frozen until analyzed.

The serum concentrations of prolactin and TSH were determined by the standard double antibody radioimmunoassay (Wakabayashi et al., 1975) with NIAMDD radioimmunoassay kits supplied by Rat Pituitary Hormone Program. The results were expressed in terms of $\mathrm{mIU}$ for prolactin and $\mathrm{mU}$ for TSH, respectively.

\section{Results}

\section{Prolaction response (Figs. 1 and 2)}

Following the repeated injection of 5 $\mu \mathrm{g} / \mathrm{kg}$ TRH at $2 \mathrm{hr}$-interval, a significant release of prolactin was shown only at the $1 \mathrm{st}$ injection in normal and TP1000A rats. In TP1000A rats, the initial level of serum prolactin was already higher than that in normal rats, as reported previously (Fujii,

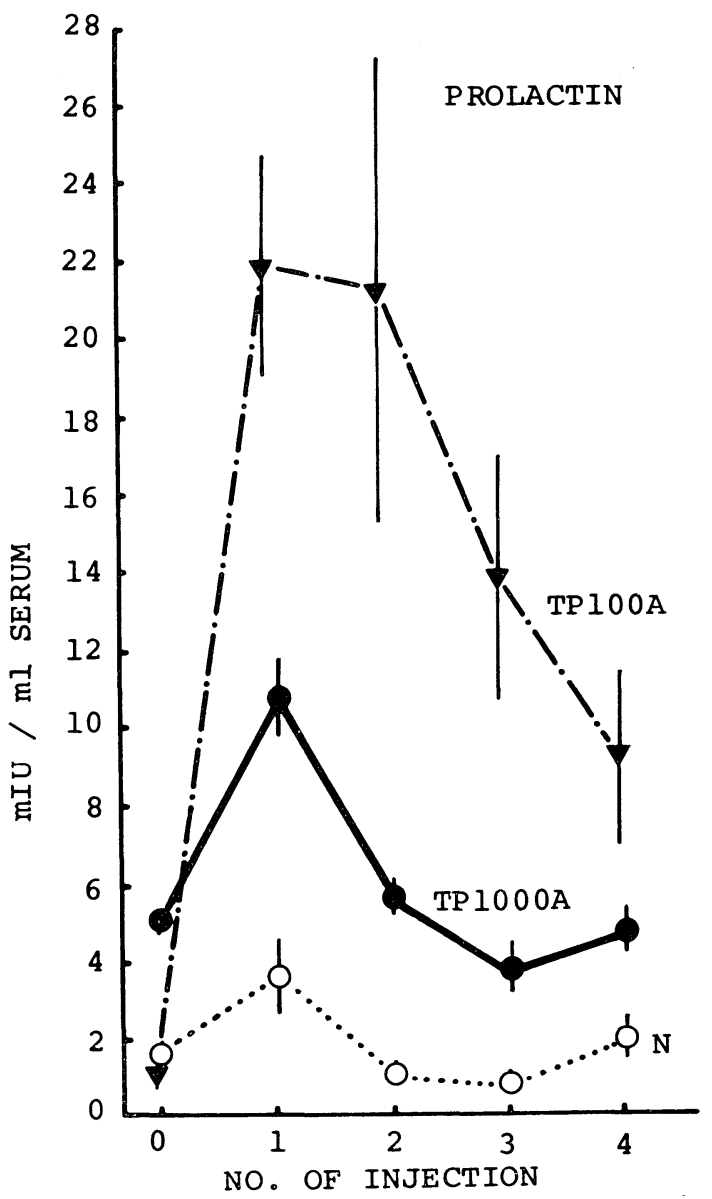

Fig. 1. Serum prolactin levels in normal (N), TP100A and TP1000A adult rats following iv injections of $5 \mu \mathrm{g} / \mathrm{kg}$ TRH every $2 \mathrm{hr}$. The rats were killed 5 min after the injection. The vertical bars represent the mean \pm SE of the mean of 4-6 rats. 
et al., 1976), and a further significant release of prolactin was shown after the 1st injection. The serum prolactin level decreased below the initial level $5 \mathrm{~min}$ after the 3rd injection of TRH, but did not decline toward the level in normal adult rats. In TP100A rats, a much greater release of prolactin by TRH was obtained at the 1 st and 2 nd injection. The maximal level reached to an average value of $21.8 \pm 2.8$ $\mathrm{mIU} / \mathrm{m} l$ serum. A significantly large amount of prolactin was released even at the $3 \mathrm{rd}$ or 4 th injection of TRH in TP100A rats.

The pituitary prolactin content in normal rats reduced significantly only after 4 injections of TRH. The basal level of prolactin in the pituitary in androgenized rats was nearly $70 \%$ higher than that in normal rats. A sharp decline was demon-

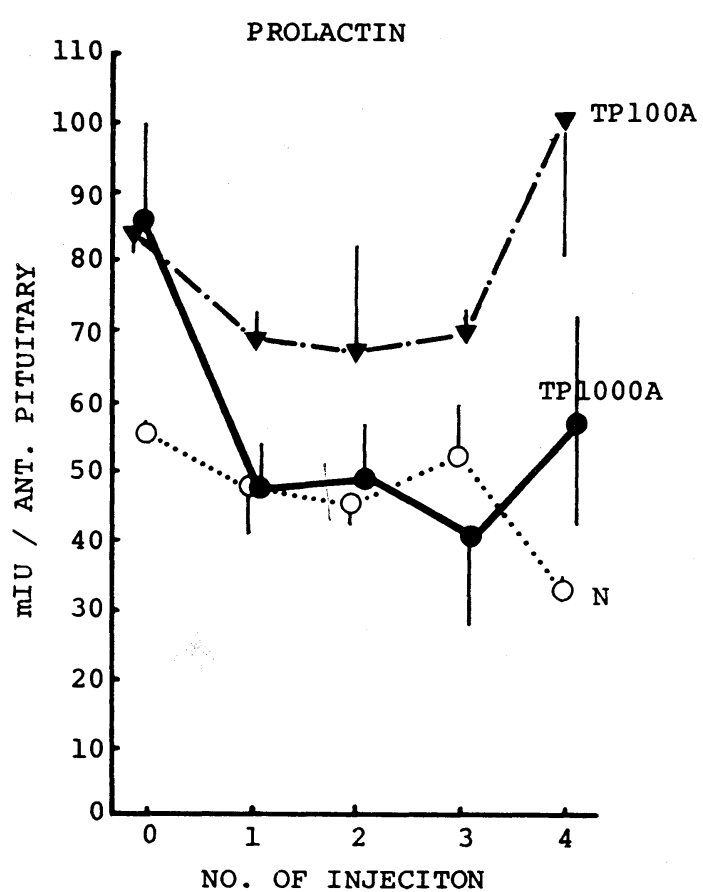

Fig. 2. Pituitary prolactin contents in normal (N), TP100A and TP1000A adult rats following iv injections of $5 \mu \mathrm{g} / \mathrm{kg}$ TRH every $2 \mathrm{hr}$. The rats were killed $5 \mathrm{~min}$ after the injection. The vertical bars represent the mean $\pm S E$ of the mean of 4-6 rats. strated in TP1000A rats after the 1 st injection and the level did not fluctuate significantly thereafter. In TP100A rats, a significant but less marked fall in the pituitary prolactin level also occurred after the 1 st injection, and the level tended to increase over the initial level after 4 injections.

\section{TSH response (Figs. 3 and 4)}

Injections of TRH produced a significant release of TSH in normal rats. A maximal response was obtained at the 1 st injection and no response was observed at the 4th injection. An increase in the serum TSH level in TP100A rats was less marked following the injection of TRH and a small but constant amount of TSH continued to be released at each injection of TRH.

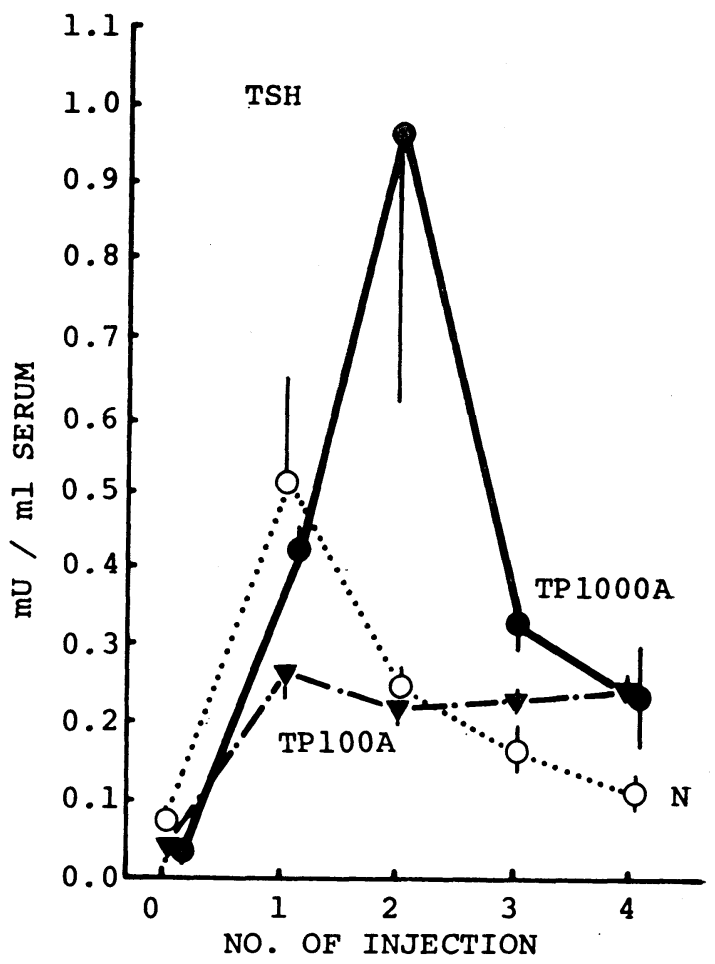

Fig. 3. Serum TSH levels in normal (N), TP100A and TP1000A adult rats following iv injections of $5 \mu \mathrm{g} / \mathrm{kg}$ TRH every $2 \mathrm{hr}$. The rats were killed 5 min later. The vertical bars represent the mean \pm SE of the mean derived from 4-6 rats. 
In TP1000A rats, the release of TSH was much greater than that seen in normal and TP100A rats. A peak appeared at the 2nd injection, though the individual variation was large.

There was no significant difference in the resting level of pituitary TSH among the three experimental groups. A significant reduction in the TSH contents was

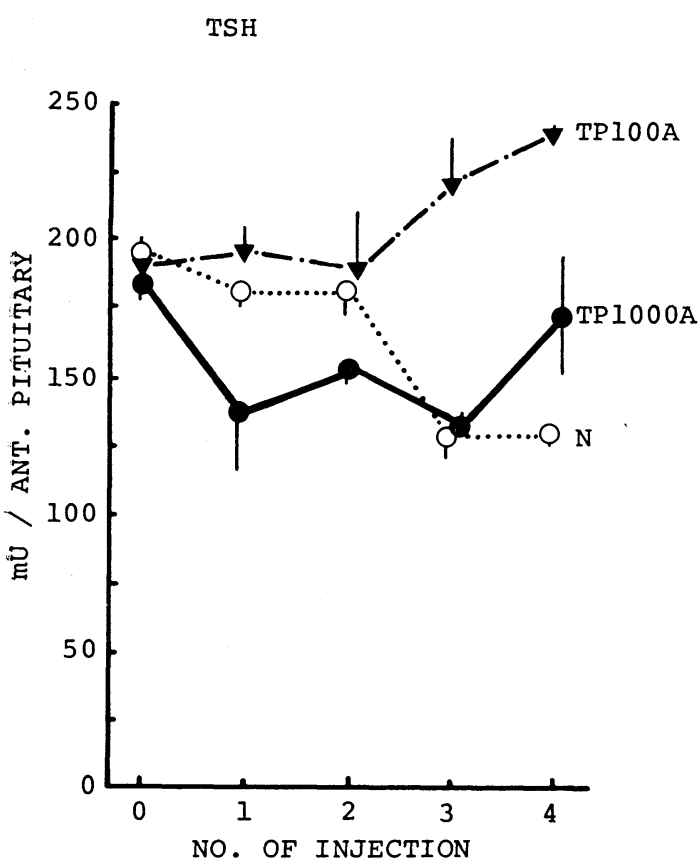

Fig. 4. Pituitary TSH contents in normal (N), TP100A and TP1000A adult rats following iv injections of $5 \mu \mathrm{g} / \mathrm{kg}$ TRH every $2 \mathrm{hr}$. The rats were killed $5 \mathrm{~min}$ later. The vertical bars represent the mean $\pm \mathrm{SE}$ of the mean derived from 4-6 rats. observed after the 3rd injection in normal rats and after the 1 st injection in TP100A rats. The TSH contents in the pituitary of TP100A rats, however, showed no significant decline but a tendency to increase after 3 injectons. The TSH contents of the pituitary after the 4 th injection tended to increase in both TP100A and TP1000A rats, but the level did not return to the initial level in TP1000A rats and increased significantly over the initial level in TP100A rats.

\section{Anterior pituitary weights}

The pituitary weights of normal and androgenized rats after repeated injections of TRH were shown in Table 1. A gradual decrease of the pituitary weight after the 2nd injection was observed in normal rats, whereas in TP1000A rats a significant decrease in the pituitary weight was noted after the 2 nd and 4 th injection. In contrast, no marked decrease but an increase was evident in TP100A rats after the 4th injection.

\section{Discussion}

The present experiments clearly indicate that not only the prolactin-producing cells (lactotrophs) but also the TSH-producing cells (thyrotrophs) in the anterior pituitary became sensitive to synthetic TRH following neonatal androgenization. The resting level of the serum prolactin in TP1000A

Table 1. Changes in the anterior pituitary weights ( $\mathrm{mg} / 100 \mathrm{~g}$ body wt.) of normal TP100A and TP1000A adult rats after injection of $5 \mu \mathrm{g} / \mathrm{kg}$ TRH at $2 \mathrm{hr}$ intervals. The rats were killed $5 \mathrm{~min}$ after injection. Each group consists of 4-6 rats.

\begin{tabular}{llcccc}
\hline \hline \multirow{2}{*}{ Group } & \multicolumn{5}{c}{ Number of injection } \\
\cline { 2 - 6 } & \multicolumn{1}{c}{0} & 1 & 2 & 3 & 4 \\
\hline Normal controls & $4.6 \pm 0.18^{\mathrm{a}}$ & $5.1 \pm 0.22$ & $4.0 \pm 0.21^{*}$ & $3.7 \pm 0.17$ & $3.4 \pm 0.18^{* *}$ \\
TP100A rats & $4.7 \pm 0.10$ & $4.9 \pm 0.10$ & $4.5 \pm 0.09$ & $4.7 \pm 0.19$ & $5.9 \pm 0.58^{* *}$ \\
TP1000A rats & $5.8 \pm 0.21$ & $6.3 \pm 0.22$ & $5.1 \pm 0.30^{*}$ & $5.5 \pm 0.68$ & $4.6 \pm 0.18^{* *}$ \\
\hline
\end{tabular}

a, Mean $\pm \mathrm{SE} ; *, \mathrm{p}<0.05$ vs. the value at 0 time; ${ }^{* *}, \mathrm{p}<0.01$ vs. the value at 0 time by Student's $t$-test. 
rats was higher than that in normal rats as reported previously (Fujii, et al., 1976) and responded to exogenous TRH to a greater extent, as compared with that in normal rats. On the other hand, the resting level of the serum prolactin in TP100A rats did not differ from that in normal female rats sacrificed on the morning of the estrous stage, though the prolacin content in the pituitary of these rats was equally high, as seen in TP1000A rats. However, the release of prolactin by exogenous TRH was the greatest and the decrease in the prolactin content in the pituitary was the smallest in TP100A rats among the three experimental groups studied. These results suggest that the prolactin synthetizing ability of lactotrophs is increased by androgenization with either of 100 or $1000 \mu \mathrm{g}$ testosterone propionate. In addition, the regulating system of prolactin release appears to be damaged by neonatal androgenization with $1000 \mu \mathrm{g}$ testosterone propionate, but unchanged or possibly with less altered when androgenized with $100 \mu \mathrm{g}$ testosterone propionate. Androgenization with 100 $\mu \mathrm{g}$ testosterone seems to induce hypersensitive state of lactotrophs to TRH.

A role of serum estrogen in enhancing the responsiveness of gonadotrophs to endogenous or exogenous LHRH has been well established by many investigators (for review see Davidson, 1969; Brown-Grant, 1971). Furthermore, prolactin secretion from the anterior pituitary of rats has been shown to be affected also by endogenous estrogen levels; a certain level of estrogen stimulates the release of prolactin (Meites and Clemens, 1972; Ratner and Peake, 1974). A predominance of circulating estrogen can be assumed in androgenized adult rats because of the persistent of vaginal estrus. In the present experiment prolactin release by synthetic TRH in androgenized adult rats is much greater than that in normal adult rats. However, LH response to synthetic LHRH in androgenized adult rats has been shown to be much lower than that in normal adult rats (Fujii, et al., 1978). Mechanisms of the increased responsiveness of gonadotrophs to exogenous LHRH in androgenized adult rats must be clarified in further experiments. An enhanced production of estradiol in the ovaries of androgenized rats during the immature period has been reported (Cheng and Johnson, 1973/74) and ballooned uterine horns were frequently seen in 3 to 4-week-old androgenized rats (Fujii, unpublished data), suggesting an increased activity of the ovary in androgenized rats during a period of very early life. Longterm effects of estrogen from an immature period in androgenized rats might be a possible cause to alter the hypothalamus which regulates the release and synthesis of prolactin in the anterior pituitary.

It is interesting to note that an increase in the TSH response to synthetic TRH was observed in TP1000A rats. The maximal release of prolactin was obtained at the 1 st injection of TRH but the maximal release of TSH was observed at the 2 nd injection in these rats. In TP100A rats, the prolactin release was the greatest among the three groups tested but TSH release was the lowest. These results might suggest that the different mechanisms are involved in the enhancement of the release of the hormones from lactotrophs and from thyrotrophs by TRH in androgenized rats.

Considerable evidence has been accumulated showing that an elevation of catecholamine activity in the hypothalamus exerts the opposite effects on TSH and prolactin release and that the inhibitory control of prolactin secretion appears to be mediated by hypothalamic dopamine (for review see Meites, 1974). The relationship between the activity of neurotransmitters in the hypothalamus and the altered responsiveness of the pituitary to exogenous TRH in androgenized rats remains to be determined. Involvement of estrogens in the regulation of TSH release by TRH has been 
suggested in intact or ovariectomized rats (De Léan et al., 1977a, b.; Steven and Lawson, 1977), A possible role of estrogen in enhancing the TSH response to TRH in androgenized rats also remains to be made clear.

The different responsiveness to the repeated administration of TRH between TP $100 \mathrm{~A}$ and TP1000A rats must be related to the different functional character of the anterior pituitary, e.g., the binding ability for TRH, the transport of hormones synthesized and/or the synthesizing ability for hormones, which might be developed during the process of androgenization in the hypothalamo-pituitary axis with the different dose of testosterone propionate.

\section{Acknowledgements}

We are grateful to Dr. A. F. Parlow, Harbor General Hospital, Torrance, California and to the National Institute of Arthritis and Metabolic Diseases, The Rat Pituitary Hormone Program, for the generous supply of radioimmunoassay kits. We are also grateful to Sankyo Pharmaceutical Co. for TRH.

\section{References}

Brown-Grant, K. Steroid Hormones and Brain Function (edited by C. H. Sawyer and R. A. Gorski) University California Press, Los Angeles and London, p. 269 (1971).

Cheng, H. C. and D. C. Johnson (1973/74). Neuroendocrinology 13, 357.

Davidson, J. M. Frontiers in Neuroendocrinology (edited by W. F. Ganong and L. Martini) Oxford University Press, London, p. 343 (1969).

De Léan, A., L. Ferland, J. Drouin, P. A. Kelly and F. Labrie (1977a). Endocrinology 100, 1496.

De Léan, A., M. Garon, P. A. Kelly and F. Labrie (1977b). ibid. 100, 1505.

Fujii, T., J. Kato and K. Wakabayashi. 22nd Annual Meeting of Eastern Branch of Japan Endocrinol. Soc. (1974).

Fujii, T., J. Kato and K. Wakabayashi (1976). Endocrinol. Japon. 23, 535.

Fujii, T., J. Kato and K. Wakabayashi (1978). ibid. $25,281$.

Jacobs, L. S., P. J. Snyder, J. F. Wilber, R. D. Utiger and W. H. Daughaday (1971). J. Clin. Endocrinol. Metab. 33, 996.

Meites, J. (1974). Excerpta Med. Intntl. Congr. Series 308, 105.

Meites, J. and J. A. Clemens (1972). Vitam. Horm. $30,165$.

Ratner, A. and G. T. Peake (1974). Proc. Soc. Exp. Biol. Med. 146, 680.

Steven, R. W. and D. W. Lawson (1977). Life Sci. 20, 261.

Tashjian, A. H., N. J. Barowsky and D. K. Jensen (1971). Biochem. Biophys. Res. Commun. 43, 516.

Wakabayashi, I., A. Arimura and A. V. Schally (1971). Proc. Soc. Exp. Biol. Med. 137, 1189.

Wakabayashi, K., P. Asai and H. Matsu-ura (1975). Gunma Symp. Endocrinol. 12, 65. 